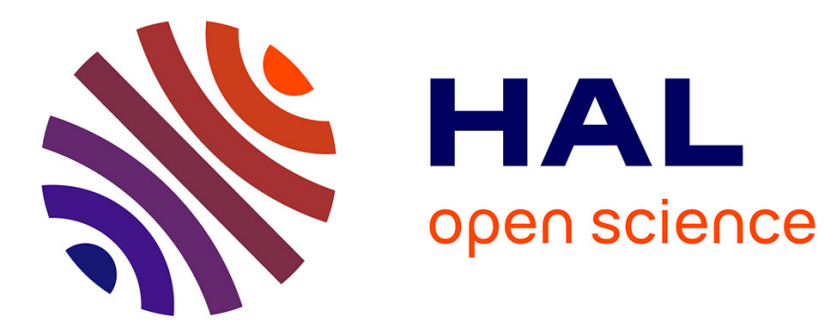

\title{
Mathematical study of very high voltage power networks II: The AC Power Flow Problem
}

\author{
J. Frederic Bonnans
}

\section{To cite this version:}

J. Frederic Bonnans. Mathematical study of very high voltage power networks II: The AC Power Flow Problem. [Research Report] RR-2544, INRIA. 1995. inria-00074134

\section{HAL Id: inria-00074134 https://hal.inria.fr/inria-00074134}

Submitted on 24 May 2006

HAL is a multi-disciplinary open access archive for the deposit and dissemination of scientific research documents, whether they are published or not. The documents may come from teaching and research institutions in France or abroad, or from public or private research centers.
L'archive ouverte pluridisciplinaire HAL, est destinée au dépôt et à la diffusion de documents scientifiques de niveau recherche, publiés ou non, émanant des établissements d'enseignement et de recherche français ou étrangers, des laboratoires publics ou privés. 
INSTITUT NATIONAL DE RECHERCHE EN INFORMATIQUE ET EN AUTOMATIQUE

\section{Mathematical study of very high voltage power networks II: The AC power flow problem}

J. Frédéric BONNANS

N 2544

Juin 1995

PROGRAMME 5 



\title{
RIN RIA
}

\section{Mathematical study of very high voltage power networks II: The AC power flow problem}

\author{
J. Frédéric BONNANS* \\ Programme 5 - Traitement du signal, automatique et productique \\ Projet Programmation mathématique \\ Rapport de recherche $\mathrm{n}^{\circ} 2544$ - Juin 1995 - 28 pages
}

\begin{abstract}
This paper is the second of a series dedicated to the mathematical study of very high voltage power networks, based on an asymptotic analysis, in which the square root of one of the small parameters is the inverse of the reference voltage of the network. We call this scheme the very high voltage approximation. We show that the active and reactive parts of the limit problem are decoupled. We give an analytic expansion for the solution of the problem, and prove the convergence of two decoupled numerical algorithm.
\end{abstract}

Key-words: Electrical networks, very high voltage, active-reactive decoupling, asymptotic analysis, sensitivity analysis, expansion of solutions, critical point, constrained optimization.

(Résumé : tsvp)

\footnotetext{
*Email: Frederic.Bonnansinria.fr. This study was supported by Electricité de France. Thanks are due to G. Blanchon and J.C. Dodu, from Direction des Etudes et Recherches, EDF, for the numerous discussions I have had with them for many years.
} 


\section{Etude mathématique des réseaux électriques de très haute tension II : Le problème du flot de puissance en courant alternatif}

Résumé : Cet article est le second d'une série consacrée à l'étude mathématique des réseaux électriques de très haute tension, basée sur une analyse asymptotique, dans laquelle la racine carrée d'un des petits paramètres est une mesure de l'inverse de la tension de référence du réseau. Ce schéma est appelé approximation des très hautes tensions. Nous obtenons un problème limite dans lequel les équations des parties actives et réactives sont découplées. Ceci permet d'obtenir le développement de la solution, ainsi que de prouver la convergence de deux algorithmes découplés.

Mots-clé : Réseaux électriques, très haute tension, découplage actif-réactif, analyse asymptotique, analyse de sensibilité, développement de solutions, point critique, optimisation sous contrainte. 
AMS Classification 26B10, 41A58, 78A99, 90B10

\section{Introduction}

The power flow problem (PFP) is, from an industrial point of view, an important issue, which involves computing the value of the voltage and power injected at the nodes of an alternating current $(\mathrm{AC})$ network. At each node, the active power and either the modulus of voltage or the reactive power are fixed. The solution must comply with Kirchhoff's and Ohm's law. This is nothing but a system of nonlinear equations; however, the highly nonlinear character of these equations makes difficult its theoretical study. What we might hope from such a study is indications about the existence and uniqueness of the solution, and the sensitivity of the solution(s) with respect to perturbations. Another useful result would be a proof of convergence of a numerical algorithm that possibly takes advantage of the specific structure of the problem. Let us quote [1] as an early reference on the mathematical study of the problem.

As PFP equations may have singularities (there may exist several, possibility degenerate solutions, or no solutions at all), it seems hopeless to give a theory that would cover all possible situations. On the other hand, the very high voltage network seems to be the most important class of applications. Henceforth, it is natural to investigate the possibility that a recognized small parameter (related to the inverse of the reference value of the voltage) allows to apply some perturbation theory.

This paper is the second in a series dedicated to the mathematical study of the optimal power flow problem (OPF), where the criterion to be minimized is the loss of energy over the network [3]. The aim of this study is to apply the recently developed perturbation theory in optimization in order to compute the expansion of OPF, and also to prove the convergence of a decoupled algorithm of resolution of OPF. A preliminary version of the results may be found in [4]. Part I of this study [5] is a preliminary step where is computed an expansion of the solution of the optimal power flow problem for direct current (DC) networks.

In this paper, we turn our attention to alternating current networks, but confine ourself to the study of PFP, because there is already much to say about the expansion of the solution of the equation of PFP without any optimization process.

The paper is organized as follows. In the next section, we set the equations of PFP, paying attention to the formulation involving explicitly the transits of active power. Section 3 presents the very high voltage approximation (VHVA). We show that the limit problem has a unique solution, which is regular and has a block-diagonal Jacobian, and compute the first term of the expansion. As a by-product of the analysis, we derive in section 4 the convergence (in the VHVA setting) of two decoupled numerical algorithms, the classical one and the one of Carpentier [7]. Networks with negligible resistances are dealt with in section 5 . The equations are shown to be those of the critical point of certain potentials. The active and reactive equations, under the constant active transits approximation, are shown to derive also from a potential. In the VHVA setting, the solutions of these critical point equations are shown to be isolated solutions of the associated optimization problems.

$\mathrm{RR} \mathrm{n}^{\circ} 2544$ 


\section{Table of notations}

$\mathcal{S}=\{1, \cdots, n\}$ set of nodes of the network.

$n=|\mathcal{S}|$ cardinal of $\mathcal{S}$.

$\bar{I}_{k \ell}$ current from node $k$ to node $l$.

$\bar{V}_{k}, V_{k}, \theta_{k}$ voltage, modulus and phase of voltage at node $k$.

$\bar{J}_{k}$ input of current at node $k$.

$j:=\sqrt{-1}$ basis of imaginary numbers.

$P_{k}, Q_{k}$ input of active and reactive power at node $k$.

$\mathcal{S}_{V}$ set of nodes over which $P$ and $V$ are given; $\mathcal{S}_{V} \neq \emptyset$.

$\mathcal{S}_{Q}$ set of nodes over which $P$ and $Q$ are given ; $\mathcal{S}_{Q}=\mathcal{S} \backslash \mathcal{S}_{V}$.

$T_{k \ell}, T_{k \ell}^{r}$ transit of active and reactive power on line $k \ell$.

$Z$ admittance matrix.

$y_{k \ell} e^{-j \xi_{k \ell}}$ admittance of inductive line from $k$ to $\ell\left(y_{k \ell} \geq 0, \xi_{k \ell} \in[\pi / 4, \pi / 2]\right)$.

$\eta_{k \ell}:=\pi / 2-\xi_{k \ell}\left(\eta_{k \ell} \in[0, \pi / 4]\right)$.

$g_{k \ell}+j h_{k \ell}$ admittance of capacitive line $\left(g_{k \ell} \geq 0, h_{k \ell} \geq 0\right)$.

$\mathbb{R}_{+}, \mathbb{R}_{++}$set of nonnegative, positive real numbers.

\section{Equations of the power flow problem}

\subsection{Basic equations}

The classical $\pi$-shaped line (see figure 1) can be decomposed in three parts: an inductive one relating nodes $k$ and $\ell$, and two capacitive ones relating $k$ and $\ell$ to the ground. Using the 


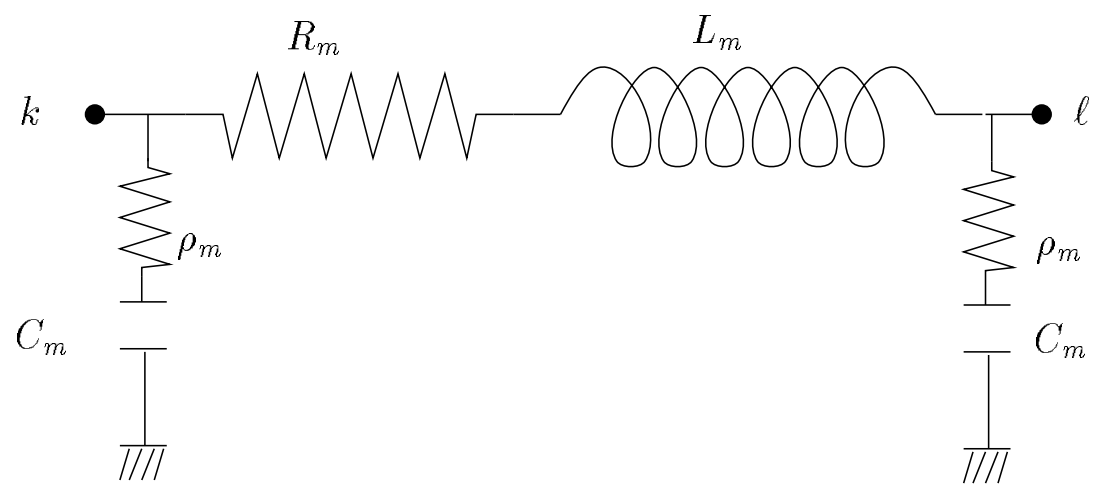

Figure 1: The $\pi$-shaped ligne

expression of admittances given in the above table, we deduce the expression of the current sent from node $k$ to node $\ell$ :

$$
\bar{I}_{k \ell}=\left(\bar{V}_{k}-\bar{V}_{\ell}\right) y_{k \ell} e^{-j \xi_{k \ell}}+\bar{V}_{k}\left(g_{k \ell}+j h_{k \ell}\right) .
$$

Note that this formulation takes in account the case when there is no line between two nodes $k$ and $\ell$ : then $y_{k \ell}=g_{k \ell}=h_{k \ell}=0$, and we may set $\xi_{k \ell}$ to $\pi / 2$. If $y_{k \ell} \neq 0$ we say that there exists an effective line between $k$ and $\ell$. Note also that in general, $\bar{I}_{k \ell}+\bar{I}_{\ell k} \neq 0$ because of the capacitive lines. We assume that the network is connected, in the sense that between any two nodes there exists a path in the graph composed of effective lines linking $k$ and $\ell$.

The transit of power from $k$ to $\ell$ is, by definition

$$
\bar{V}_{k} \bar{I}_{k \ell}^{*}=V_{k}\left(V_{k}-V_{\ell} e^{j\left(\theta_{k}-\theta_{\ell}\right)}\right) y_{k \ell} e^{j \xi_{k \ell}}+V_{k}^{2}\left(g_{k \ell}-j h_{k \ell}\right) .
$$

Its active et reactive parts, called the transit of active and reactive power from $k$ to $\ell$, are

$$
\begin{aligned}
& T_{k \ell}:=V_{k}^{2}\left(y_{k \ell} \cos \xi_{k \ell}+g_{k \ell}\right)-y_{k \ell} V_{k} V_{\ell} \cos \left(\theta_{k}-\theta_{\ell}+\xi_{k \ell}\right), \\
& T_{k \ell}^{r}:=V_{k}^{2}\left(y_{k \ell} \sin \xi_{k \ell}-h_{k \ell}\right)-y_{k \ell} V_{k} V_{\ell} \sin \left(\theta_{k}-\theta_{\ell}+\xi_{k \ell}\right) .
\end{aligned}
$$

As the resistance of the inductive line is usually small, the value of $\xi_{k \ell}$ is slightly inferior to $\pi / 2$. Therefore it is useful to make the change of parameter

$$
\xi_{k \ell}=\pi / 2-\eta_{k \ell},
$$

where $\eta_{k \ell}$ is small and nonnegative. The expression of the transits in term of $\eta_{k \ell}$ are 


$$
\begin{aligned}
& T_{k \ell}:=V_{k}^{2}\left(y_{k \ell} \sin \eta_{k \ell}+g_{k \ell}\right)+y_{k \ell} V_{k} V_{\ell} \sin \left(\theta_{k}-\theta_{\ell}-\eta_{k \ell}\right), \\
& T_{k \ell}^{r}:=V_{k}^{2}\left(y_{k \ell} \cos \eta_{k \ell}-h_{k \ell}\right)-y_{k \ell} V_{k} V_{\ell} \cos \left(\theta_{k}-\theta_{\ell}-\eta_{k \ell}\right) .
\end{aligned}
$$

Taking in account Kirchhoff's law:

$$
\bar{J}_{k}=\sum_{\ell \neq k} \bar{I}_{k \ell}
$$

we see that the amount of active power and reactive power, respectively, injected at node $k$ are equal to the sum of transits of active and reactive power at lines starting from node $k$ :

$$
\mathcal{R} e\left(\bar{V}_{k} \bar{J}_{k}^{*}\right)=\sum_{\ell \neq k} T_{k \ell} ; \text { resp. } \mathcal{I} m\left(\bar{V}_{k} \bar{J}_{k}^{*}\right)=\sum_{\ell \neq k} T_{k \ell}^{r} .
$$

Let $\left(\mathcal{S}_{V}, \mathcal{S}_{Q}\right)$ be a partition of $\mathcal{S}$; i.e., $\mathcal{S}_{V}$ and $\mathcal{S}_{Q}$ are subsets of $\mathcal{S}$ such that any $k \in \mathcal{S}$ belongs either to $\mathcal{S}_{V}$ or $\mathcal{S}_{Q}$, but not both. We assume that $\mathcal{S}_{V} \neq \emptyset$. Let a be a n-dimensional vector such that

$$
\sum_{k \in \mathcal{S}} a_{k}=1
$$

This paper is devoted to the study of the following problem: to solve w.r.t. $(\omega, \theta, V)$, with $\omega$ a scalar parameter, the following system of equations:

$$
\begin{aligned}
\omega a+\sum_{\ell \neq k} T_{k \ell} & =P_{k}, k \in \mathcal{S}, \\
a^{t} \theta & =0, \\
\sum_{\ell \neq k} T_{k \ell}^{r} & =Q_{k}, k \in \mathcal{S}_{Q}, \\
V & =V^{D} \text { on } \mathcal{S}_{V} .
\end{aligned}
$$

Here the right hand side of the equations is given, $V^{D}$ has positive components, and $T_{k \ell}$ and $T_{k \ell}^{r}$ satisfy (4) and (5). Substituting these expressions and dividing the reactive power balance by the value of voltages, we obtain

$$
\begin{aligned}
\omega a+\sum_{\ell \neq k} V_{k}^{2}\left(y_{k \ell} \sin \eta_{k \ell}+g_{k \ell}\right)+y_{k \ell} V_{k} V_{\ell} \sin \left(\theta_{k}-\theta_{\ell}-\eta_{k \ell}\right) & =P_{k}, k \in \mathcal{S}, \\
a^{t} \theta & =0 \\
\sum_{\ell \neq k} V_{k}\left(y_{k \ell} \cos \eta_{k \ell}-h_{k \ell}\right)-y_{k \ell} V_{\ell} \cos \left(\theta_{k}-\theta_{\ell}-\eta_{k \ell}\right) & =\frac{Q_{k}}{V_{k}}, k \in \mathcal{S}_{Q}, \\
V & =V^{D} \text { on } \mathcal{S}_{V} .
\end{aligned}
$$


(Note that the above sums apply to the expression until the equality symbol; we do not write brackets in order to avoid heavy notation. No confusion is possible).

The term $\omega a$ is called the external source of active power. This term is necessary, as we will see later, in order to check (in some cases) that the above system is well-posed. The power flow equations being invariant w.r.t. a tranlation of phases, the purpose of the second constraint is to recover a unique solution. We could have instead fixed the phase at a certain node, e.g. like $\theta_{1}=0$. However, writing $a^{t} \theta=0$ makes easier the analysis of these equations. Finally, note that as we want to study networks with high values of voltages at all nodes, there is no harm in dividing the reactive power flow equations by the value of voltages. We call active (resp. reactive) part of this system the two first (resp. last) equations.

We assume throughout the paper that the parameters $(\varepsilon, \eta, g, h)$ satisfy

$$
(\varepsilon, \eta, g, h) \in A:=\mathbb{R}_{+} \times[0, \pi / 4]^{n} \times \mathbb{R}_{+}^{n} \times \mathbb{R}_{+}^{n} .
$$

We search for a solution of the power equation in the following set:

$$
(\omega, \theta, V) \in E:=\mathbb{R} \times \Theta \times \mathcal{V},
$$

where

$$
\Theta:=\left\{\theta \in \mathbb{R}^{n} ; \theta_{k}-\theta_{\ell} \in[-\pi / 4, \pi / 4] ; \forall k, \ell \in \mathcal{S}\right\}
$$

and

$$
\mathcal{V}=\left\{V \in \mathbb{R}^{n} ; V_{k}>0, \forall k=1, \ldots, n\right\} .
$$

The above relations imply

$$
\theta_{k}-\theta_{\ell}-\eta_{k \ell} \in[-\pi / 2, \pi / 2], \forall k, \ell \in \mathcal{S} .
$$

Remark: Actually it suffices to restrict $\theta_{k}-\theta_{\ell}$ to $[-\pi / 4, \pi / 4]$ whenever $y_{k \ell}>0$, because otherwise $\theta_{k}-\theta_{\ell}$ does never appear in the power flow equations. We keep the above definition of $\Theta$ for the sake of simplicity.

\subsection{Other expressions using active transits}

Motivated by the fact that the formulations using active transits seem to be well suited for numerical computations [7], we state such a formulation. From (4), we get

$$
\sin \left(\theta_{k}-\theta_{\ell}-\eta_{k \ell}\right)=\left[T_{k \ell}-V_{k}^{2}\left(y_{k \ell} \sin \eta_{k \ell}+g_{k \ell}\right)\right] / y_{k \ell} V_{k} V_{\ell} .
$$

As observed above, we have $\left(\theta_{k}-\theta_{\ell}-\eta_{k \ell}\right) \in[-\pi / 2, \pi / 2]$ for all $k, \ell \in \mathcal{S}$. It follows that

$$
\cos \left(\theta_{k}-\theta_{\ell}-\eta_{k \ell}\right)=\sqrt{1-\sin \left(\theta_{k}-\theta_{\ell}-\eta_{k \ell}\right)^{2}} .
$$

Combining the two identities with (5), we get the following relation:

$$
T_{k \ell}^{r}=V_{k}^{2}\left(y_{k \ell} \cos \eta_{k \ell}-h_{k \ell}\right)-y_{k \ell} V_{k} V_{\ell} \sqrt{1-\frac{\left(T_{k \ell}-V_{k}^{2}\left(y_{k \ell} \sin \eta_{k \ell}+g_{k \ell}\right)\right)^{2}}{\left(y_{k \ell} V_{k} V_{\ell}\right)^{2}}} .
$$

$\mathrm{RR} \mathrm{n}^{\circ} 2544$ 
This leads to an alternating formulation of the power flow problem that we call active transits formulation:

$$
\begin{aligned}
T_{k \ell}-V_{k}^{2}\left(y_{k \ell} \sin \eta_{k \ell}+g_{k \ell}\right)-y_{k \ell} V_{k} V_{\ell} \sin \left(\theta_{k}-\theta_{\ell}-\eta_{k \ell}\right) & =0, k, \ell \in \mathcal{S}, \\
\omega a+\sum_{\ell \neq k} T_{k \ell} & =P_{k}, k \in \mathcal{S}, \\
a^{t} \theta & =0, \\
\sum_{\ell \neq k} V_{k}\left(y_{k \ell} \cos \eta_{k \ell}-h_{k \ell}\right)-y_{k \ell} V_{\ell} \sqrt{1-\frac{\left(T_{k \ell}-V_{k}^{2}\left(y_{k \ell} \sin \eta_{k \ell}+g_{k \ell}\right)\right)^{2}}{\left(y_{k \ell} V_{k} V_{\ell}\right)^{2}}} & =\frac{Q_{k}}{V_{k}}, k \in \mathcal{S}_{Q}, \\
V & =V^{D} \text { on } \mathcal{S}_{V},
\end{aligned}
$$

where the set of unknowns is now $(T, \omega, \theta, V)$. We call active (resp. reactive) part of this system the three first (resp. two last) equations.

Remark Instead of the transit of active power themself, we may, following a suggestion by [3], use the average value of active transits over the line from $k$ to $\ell$ :

$$
\tau_{k \ell}:=\frac{1}{2}\left(T_{k \ell}-T_{\ell k}\right)
$$

The expression of $\tau_{k \ell}$ w.r.t. $V$ and $\theta$ is, by (4) :

$$
\tau_{k \ell}=\frac{1}{2}\left(V_{k}^{2}-V_{\ell}^{2}\right)\left(y_{k \ell} \sin \eta_{k \ell}+g_{k \ell}\right)+y_{k \ell} V_{k} V_{\ell} \cos \eta_{k \ell} \sin \left(\theta_{k}-\theta_{\ell}\right) .
$$

Using this, we can express $\theta_{k}-\theta_{\ell}$ as a function of $\tau_{k \ell}, V$ and $\theta$. Substituting this expression in the reactive power flow equations, we obtain another equivalent formulation. For the sake of simplicity, we confine ourself to the active transits formulation above.

\subsection{Losses of active power}

Because the voltages are set usually so as to keep small the loss of active power over the lines, it is of interest to compute the expression of this amount. The loss on line $k \ell$ is

$$
\begin{aligned}
\pi_{k \ell} & :=T_{k \ell}+T_{\ell k}, \\
& =\left(V_{k}^{2}+V_{\ell}^{2}\right)\left(y_{k \ell} \sin \eta_{k \ell}+g_{k \ell}\right)+y_{k \ell} V_{k} V_{\ell}\left[\sin \left(\theta_{k}-\theta_{\ell}-\eta_{k \ell}\right)+\sin \left(\theta_{\ell}-\theta_{k}-\eta_{k \ell}\right)\right], \\
& =\left(V_{k}^{2}+V_{\ell}^{2}\right)\left(y_{k \ell} \sin \eta_{k \ell}+g_{k \ell}\right)-2 y_{k \ell} V_{k} V_{\ell} \sin \eta_{k \ell} \cos \left(\theta_{k}-\theta_{\ell}\right) .
\end{aligned}
$$

We may write the equivalent expression

$$
\pi_{k \ell}=g_{k \ell}\left(V_{k}^{2}+V_{\ell}^{2}\right)+y_{k \ell}\left(V_{k}-V_{\ell}\right)^{2} \sin \eta_{k \ell}+2 y_{k \ell} V_{k} V_{\ell}\left(1-\cos \left(\theta_{k}-\theta_{\ell}\right)\right) \sin \eta_{k \ell} .
$$

This allows to check that $\pi_{k \ell} \geq 0$ (as $g_{k \ell} \geq 0, y_{k \ell} \geq 0$, and $\eta_{k \ell} \in[0, \pi / 4]$ by hypothesis), i.e., as is expected, the loss of active power cannot be negative. The terms on the right-hand 
side may be interpretated as the loss of power over the capacitive line, a term related to the difference of voltages but not to phases, and a last term primarily related to the difference of phases. If $g=0$ and $\eta=0$, there is no resistance on the network, and therefore no loss of active power.

\section{The very high voltage approximation}

\subsection{Principle}

We start from system (6)-(9), but consider a family of problems in which, instead of a single given voltage $V^{D}$, we have a curve $V^{D} / \sqrt{\varepsilon}$, where $\varepsilon$ is a small positive parameter. The resulting system, in which $T_{k \ell}$ and $T_{k \ell}^{r}$ satisfy equations (4) and (5) is

$$
\begin{aligned}
\omega a+\sum_{\ell \neq k} T_{k \ell} & =P_{k}, k \in \mathcal{S}, \\
a^{t} \theta & =0, \\
\sum_{\ell \neq k} T_{k \ell}^{r} & =Q_{k}, k \in \mathcal{S}_{Q}, \\
V & =V^{D} / \sqrt{\varepsilon} \text { on } \mathcal{S}_{V} .
\end{aligned}
$$

We scale the voltage and the source of active power by making the change of variable

$$
\hat{V}:=\sqrt{\varepsilon} V \text { and } \hat{\omega}:=\varepsilon \omega .
$$

After some simple algebra using (4) and (5), we get the following system:

$$
\begin{aligned}
\hat{\omega} a+\sum_{\ell \neq k} \hat{V}_{k}^{2}\left(y_{k \ell} \sin \eta_{k \ell}+g_{k \ell}\right)+y_{k \ell} \hat{V}_{k} \hat{V}_{\ell} \sin \left(\theta_{k}-\theta_{\ell}-\eta_{k \ell}\right) & =\varepsilon P_{k}, k \in \mathcal{S}, \\
a^{t} \theta & =0 \\
\sum_{\ell \neq k} \hat{V}_{k}\left(y_{k \ell} \cos \eta_{k \ell}-h_{k \ell}\right)-y_{k \ell} \hat{V}_{\ell} \cos \left(\theta_{k}-\theta_{\ell}-\eta_{k \ell}\right) & =\varepsilon \frac{Q_{k}}{\hat{V}_{k}}, k \in \mathcal{S}_{Q}, \\
\hat{V} & =V^{D} \text { on } \mathcal{S}_{V} .
\end{aligned}
$$

This system is similar to the original power flow equations (6)-(9), except for the coefficient $\varepsilon$ multiplying $P$ and $Q$.

\subsection{The limit problem}

We choose to consider as small parameter the set

$$
\alpha:=\{\varepsilon, \eta, g, h\} \in A .
$$

$\mathrm{RR} \mathrm{n}^{\circ} 2544$ 
This is a classical hypothesis in the field of high voltage electrical networks, see [9]. Note that we do not make any hypothesis on the solution of the power equation, but only on the data (in particular, that the difference of phases is small will be a result of the analysis and not an hypothesis). The parameter $\varepsilon$ measures the order of magnitude of the reference value of the voltage of the network. Considering $\eta$ as small amounts to say that the real part of the inductive line is small w.r.t. its imaginary part. Neglecting $g$ and $h$ amounts to neglect the capacitive lines.

Setting the small parameter $\alpha$ to 0 in (15)-(18), we get the limit system

$$
\begin{aligned}
\hat{\omega} a+\sum_{\ell \neq k} y_{k \ell} \hat{V}_{k} \hat{V}_{\ell} \sin \left(\theta_{k}-\theta_{\ell}\right) & =0, k \in \mathcal{S}, \\
a^{t} \theta & =0, \\
\sum_{\ell \neq k} y_{k \ell}\left[\hat{V}_{k}-\hat{V}_{\ell} \cos \left(\theta_{k}-\theta_{\ell}\right)\right] & =0, k \in \mathcal{S}, \\
\hat{V} & =V^{D} \text { on } \mathcal{S}_{V} .
\end{aligned}
$$

We check that this limit problem has a simple decoupled structure. A solution of a system of nonlinear equations is said to be regular whenever the associated Jacobian is invertible. Regularity is a cornerstone of perturbation analysis as well as of the analysis of algorithms. We say that the active and reactive parts of the Jacobian are decoupled if the Jacobian is block-diagonal, the linearization of the active (resp. reactive) part not involving the variation of voltages (resp. source of external power and phases). We remind that the set $E$ (where we look for solutions of the power equation) was defined in section 2.1.

Lemma 1 The limit system has a unique solution in E, namely

$$
\hat{\omega}^{0}=0, \quad \theta^{0}=0, \quad \hat{V}^{0},
$$

where $\hat{V}^{0} \in \mathbb{R}_{++}^{n}$ is solution of the $D C$ linear equation

$$
\hat{V}=V^{D} \text { on } \mathcal{S}_{V} ; \sum_{\ell \neq k} y_{k \ell}\left(\hat{V}_{k}-\hat{V}_{\ell}\right)=0, k \in \mathcal{S}_{P} .
$$

In addition, this solution is regular and the active and reactive parts of the associated Jacobian are decoupled.

Proof Let $(\hat{\omega}, \theta, \hat{V})$ be solution of (19)-(22). Summing the active power equations over $\mathcal{S}$, and noticing that the active transit from $k$ to $\ell$ for the limit problem is

$$
T_{k \ell}=y_{k \ell} \hat{V}_{k} \hat{V}_{\ell} \sin \left(\theta_{k}-\theta_{\ell}\right)=-T_{\ell k},
$$

we find that

$$
\hat{\omega}=\hat{\omega} \sum_{k \in \mathcal{S}} a_{k}=-\sum_{k, \ell \in \mathcal{S}} T_{k \ell}=0
$$


whence $\hat{\omega}=0$. Let $k$ be a node at which $\theta$ attains its maximum; then

$$
\sum_{\ell \neq k} y_{k \ell} \hat{V}_{k} \hat{V}_{\ell} \sin \left(\theta_{k}-\theta_{\ell}\right)=0
$$

Since $\theta \in \Theta$, we have $\sin \left(\theta_{k}-\theta_{\ell}\right) \geq 0$ over $\mathcal{S}$. Therefore, $\theta$ has the same value at all neighbouring nodes (i.e. nodes connected by an effective line). The network being connected, by an induction argument it follows that $\theta$ has the same value over all $\mathcal{S}$. As $a^{t} \theta=0$, a null value of $\theta$ over $\mathcal{S}$ is the only way to satisfy the active power equation.

Considering now the reactive power equations, we find that $\hat{V}$ is solution of the linear system stated above, which is nothing but the equation of a linear DC problem, where $V$ is given at $\mathcal{S}_{V} \neq \emptyset$ and the injection of current at all other nodes is null: the network being connected, this problem has a unique solution with positive components.

We check that this solution is regular: the linearized reactive power equation is the equation of a linear DC problem, which is a well-posed linear system. The linearized active power equations, denoting by $\left(d \hat{\omega}, d \theta, d P, d \theta^{0}\right)$ the linearized variables and the right-handside, are

$$
\left\{\begin{array}{l}
(d \hat{\omega}) a+\sum_{\ell \neq k} y_{k \ell} \hat{V}_{k}^{0} \hat{V}_{\ell}^{0}\left(d \theta_{k}-d \theta_{\ell}\right)=d P_{k}, k \in \mathcal{S} ; \\
a^{T} d \theta=d \theta^{0} .
\end{array}\right.
$$

Summing over $\mathcal{S}$, we find that $d \omega=\sum_{k \in \mathcal{S}} d P_{k}$, while $d \theta$ is solution of

$$
\begin{aligned}
& \sum_{\ell \neq k} y_{k \ell} \hat{V}_{k}^{0} \hat{V}_{\ell}^{0}\left(d \theta_{k}-d \theta_{\ell}\right)=d P_{k}-(d \omega) a, k \in \mathcal{S}, \\
& a^{T} d \theta=0,
\end{aligned}
$$

which is also a linear DC problem with given positive resistances over effective lines and injection of current at all nodes, the sum of injected currents being null, and a normalizing constraint. The network being connected, this problem has also a unique solution.

The next statement deals with the convergence of solutions of (15)-(18) when $\alpha \rightarrow 0$ in $A$. In order to pass to the limit in the equation we must exclude the case when inf $V_{k} \rightarrow 0$. By lemma 1, the solution of the limit equation satisfies

$$
\min _{k \in \mathcal{S}} V_{k}^{0}=\min _{k \in \mathcal{S}_{V}} V_{k}^{0}=\min V^{D}>0 .
$$

Therefore, we may restrict $V$ to the set

$$
\mathcal{V}_{\beta}:=\left\{V \in \mathbb{R}^{n} ; V_{k} \geq \beta, \forall k \in \mathcal{S}\right\},
$$

with

$$
0<\beta<\min V^{D}
$$

In summary, we search for $(\omega, \Theta, V)$ in the set (remind that $\Theta$ was defined in point 2.1)

$$
E_{\beta}:=\mathbb{R} \times \Theta \times \mathcal{V}_{\beta} .
$$

$\operatorname{RR} n^{\circ} 2544$ 
Theorem 1 Fix $\beta$ as above. Then, for $\alpha$ close enough to 0 in $A$, there exists a unique solution $(\hat{\omega}, \theta, \hat{V})$ of $(15)-(18)$ in $E_{\beta}$, whose dependence with respect to $\alpha$ is analytical.

Proof The proof is in two parts. We first prove that for $\alpha$ in a certain neighborhood of 0 , any solution of the power flow equations remains in a bounded set. Passing to the limit in the power equation, it follows that these solutions (if they exist) converge to the unique solution of the limit problem. Then we conclude by applying the implicit function theorem (IFT) to the limit problem.

Proceeding by contradiction, let us assume that there exists an unbounded sequence of solutions to the power flow problem, for values of $\alpha$ converging to 0 in $A$. We may write the expression of transit of reactive power (5), in which we write $\hat{V}_{k}, \hat{V}_{\ell}$ instead of $V_{k}$ and $V_{\ell}$, as

$$
y_{k \ell}\left[1-\frac{\hat{V}_{\ell}}{\hat{V}_{k}} \cos \left(\theta_{k}-\theta_{\ell}\right)\right] \cos \eta_{k \ell}=\frac{T_{k \ell}^{r}}{\hat{V}_{k}^{2}}+h_{k \ell}+y_{k \ell} \frac{\hat{V}_{\ell}}{\hat{V}_{k}} \sin \left(\theta_{k}-\theta_{\ell}\right) \sin \eta_{k \ell} .
$$

By (17), $\sum_{k \neq \ell} T_{k \ell}^{r} / \hat{V}_{k}^{2}=\varepsilon Q_{k} / \hat{V}_{k}^{2}=o\left(1 / \hat{V}_{k}^{2}\right)$. Therefore when $\alpha \rightarrow 0$, and $V \in \mathcal{V}_{\beta}$ :

$$
\sum_{\ell \neq k} y_{k \ell}\left[1-\frac{\hat{V}_{\ell}}{\hat{V}_{k}} \cos \left(\theta_{k}-\theta_{\ell}\right)\right] \cos \eta_{k \ell}=o\left(\frac{\hat{V}_{\ell}}{\hat{V}_{k}}\right)+o(1)
$$

Extracting if necessary a subsequence, we may assume that there exists $\hat{\mathcal{S}} \subset \mathcal{S}_{Q}$ such that

$$
\frac{1}{V_{k}} \max _{\ell \in \mathcal{S}} V_{\ell} \rightarrow 1 \text { iff } V_{k} \in \hat{\mathcal{S}}
$$

Pick $k \in \hat{\mathcal{S}}$. Then the r.h.s. of $(24)$ is $o(1)$. Each term of the sum in the l.h.s. is bounded, and $\eta_{k \ell} \downarrow 0$, whence

$$
\sum_{\ell} y_{k \ell}\left[1-\frac{\hat{V}_{\ell}}{\hat{V}_{k}} \cos \left(\theta_{k}-\theta_{\ell}\right)\right]=o(1)
$$

By definition of $\hat{\mathcal{S}}$, we have

$$
\frac{\hat{V}_{\ell}}{\hat{V}_{k}} \leq 1+o(1)
$$

As $\cos \left(\theta_{k}-\theta_{\ell}\right) \leq 1$, we deduce that $\hat{V}_{\ell} / \hat{V}_{k} \rightarrow 1$ whenever $\ell$ is a neighbor of $k$, i.e. $\hat{\mathcal{S}}$ contains all its neighboring nodes. However, no node of $\mathcal{S}_{V}$ is included in $\hat{\mathcal{S}}$, as $\hat{V}=V^{D}$ is bounded on $\mathcal{S}_{V}$. The network being connected, we arrive to the desired contradiction.

So we have proved that for $\alpha$ close to 0 , any solution of the power flow equations remains in a bounded set. Passing to the limit in the power flow equations, we conclude that these solutions have no other limit-point than $\left(\hat{\omega}^{0}, \theta^{0}, \hat{V}^{0}\right)$.

It remains to perform a local study, in an arbitrarily small neighborhood of $\left(\hat{\omega}^{0}, \theta^{0}, \hat{V}^{0}\right)$. There we can use the implicit function theorem for analytical function, see e.g. [10], from which the conclusion follows. 


\subsection{Expansion of the solution}

In order to compute the first-order term of the expansion, we write the linearized system associated with (15)-(18). Its active and reactive parts are of course decoupled:

$$
\left\{\begin{aligned}
(d \hat{\omega}) a+\sum_{\ell \neq k}\left(\hat{V}_{k}^{0}\right)^{2}\left(y_{k \ell} \eta_{k \ell}+g_{k \ell}\right)+y_{k \ell} \hat{V}_{k}^{0} \hat{V}_{\ell}^{0}\left(d \theta_{k}-d \theta_{\ell}-\eta_{k \ell}\right) & =\varepsilon P_{k}, \quad k \in \mathcal{S}, \\
a^{t} d \theta & =0,
\end{aligned}\right.
$$

and

$$
\left\{\begin{aligned}
\sum_{\ell \neq k} y_{k \ell}\left(d \hat{V}_{k}-d \hat{V}_{\ell}\right) & =\varepsilon \frac{Q_{k}}{\hat{V}_{k}^{0}}+\hat{V}_{k}^{0} \sum_{\ell \neq k} h_{k \ell}, \quad k \in \mathcal{S}_{Q}, \\
d \hat{V} & =0 \text { on } \mathcal{S}_{V},
\end{aligned}\right.
$$

and we observe that $d \hat{V}$ is a function of $(\varepsilon, h)$, whereas $(d \hat{\omega}, d \theta)$ depends on $(\varepsilon, g, \eta)$. As observed before, each of the two systems above may be interpretated in term of direct current equations. Summing the active power equations over the network, and using $\sum_{k} a_{k}=1$, we get an explicit formula for $d \hat{\omega}$ :

$$
d \hat{\omega}=\varepsilon \sum_{k \in \mathcal{S}} P_{k}-\sum_{k>\ell} g_{k \ell}\left[\left(\hat{V}_{k}^{0}\right)^{2}+\left(\hat{V}_{\ell}^{0}\right)^{2}\right]-\sum_{k>\ell} y_{k \ell} \eta_{k \ell}\left(\hat{V}_{k}^{0}-\hat{V}_{\ell}^{0}\right)^{2} .
$$

Let us now express these results in term of the the physical variables

$$
V=\hat{V} / \sqrt{\varepsilon}, \quad \omega=\hat{\omega} / \varepsilon .
$$

Denoting by $(d \hat{\omega}, d \theta, d \hat{V})$ the solution of $(25)(26)$ and using the fact that $d \hat{\omega}=O(\varepsilon+$ $\|g\|+\|\eta\|), d \theta=O(\varepsilon+\|g\|+\|\eta\|), d V=O(\varepsilon+\|h\|)$, we obtain

$$
\left\{\begin{aligned}
\omega^{\varepsilon} & =\frac{1}{\varepsilon} d \hat{\omega}+O\left(\frac{\|\alpha\|^{2}}{\varepsilon}\right)=O\left(1+\frac{\|g\|+\|\eta\|+\|h\|^{2}}{\varepsilon}\right) \\
\theta^{\varepsilon} & =d \theta+O\left(\|\alpha\|^{2}\right)=O(\varepsilon+\|g\|+\|\eta\|)+O\left(\|h\|^{2}\right) \\
V^{\varepsilon} & =\frac{1}{\sqrt{\varepsilon}}\left[\hat{V}^{0}+d \hat{V}+O\left(\|\alpha\|^{2}\right)\right]=O\left(\frac{1}{\sqrt{\varepsilon}}\right) .
\end{aligned}\right.
$$

As $\omega^{\varepsilon}$ is the external source of active power, the total losses over the network will remain bounded when $\alpha \rightarrow 0$ iff $\omega^{\varepsilon}$ remains itself bounded, i.e. if $\|g\|+\|\eta\|+\|h\|^{2}=O(\varepsilon)$. We recover this result by analysing the loss of active power on line $k \ell$. Using subsection 2.3 , we get

$$
\begin{aligned}
\pi_{k \ell}= & \frac{g_{k \ell}}{\varepsilon}\left[\left(\hat{V}_{k}^{0}\right)^{2}+\left(\hat{V}_{\ell}^{0}\right)^{2}\right]+\frac{y_{k \ell} \eta_{k \ell}}{\varepsilon}\left(\hat{V}_{k}^{0}-\hat{V}_{\ell}^{0}\right)^{2} \\
& +2 \frac{g_{k \ell}}{\varepsilon}\left(\hat{V}_{k}^{0} d \hat{V}_{k}^{0}+\hat{V}_{\ell}^{0} d \hat{V}_{\ell}^{0}\right)+2 \frac{y_{k \ell} \eta_{k \ell}}{\varepsilon}\left(\hat{V}_{k}^{0}-\hat{V}_{\ell}^{0}\right)\left(d \hat{V}_{k}-d \hat{V}_{\ell}\right)+\frac{1}{\varepsilon} O\left(\|\alpha\|^{3}\right) .
\end{aligned}
$$


Remark From the above formulas we get some useful information for the design of the network, for instance if we consider the external source of power as a scarce resource. In that case, we may first compute $\hat{V}^{0}$ (that does not depend on $P$ and $Q$ ) as then $\operatorname{design} P_{k}$ so as to get $d \hat{\omega}=0$, i.e.

$$
\varepsilon \sum_{k \in \mathcal{S}} P_{k}=\sum_{k>\ell} g_{k \ell}\left[\left(\hat{V}_{k}^{0}\right)^{2}+\left(\hat{V}_{\ell}^{0}\right)^{2}\right]+\sum_{k>\ell} y_{k \ell} \eta_{k \ell}\left(\hat{V}_{k}^{0}-\hat{V}_{\ell}^{0}\right)^{2} .
$$

Similarly, if we want to keep the variation of voltage close to 0 , then we may take the value of $Q_{k}$ close to the ideal value

$$
Q_{k}=-\frac{1}{\varepsilon}\left(\hat{V}_{k}^{0}\right)^{2} \sum_{\ell \neq k} h_{k \ell}, k \in \mathcal{S}_{Q}
$$

because if this equality holds, then $d \hat{V}=0$. In practice, this may lead to large negative values, whereas the reactive power value at node $k$ may be choosed in a certain interval of $\mathbb{R}$, say $\left[\underline{Q}_{k}, \bar{Q}^{k}\right]$. If we want to keep small the variations of voltage over the network, a possible heuristic is to take for $Q_{k}$ at each node a value in $\left[\underline{Q}_{k}, \bar{Q}^{k}\right]$ closest to the ideal value, i.e.

$$
Q_{k}=\max \left(\underline{Q}_{k}, \min \left(\bar{Q}^{k},-\frac{1}{\varepsilon}\left(\hat{V}_{k}^{0}\right)^{2} \sum_{\ell \neq k} h_{k \ell}\right)\right), k \in \mathcal{S}_{Q} .
$$

Another possibility is to solve a simple linear least-square problem such as

$$
\operatorname{Min}_{d Q, d V} \sum_{k \in \mathcal{S}_{Q}}\left(d V_{k}\right)^{2} \text { s.t. }(26), Q_{k} \in\left[\underline{Q}_{k}, \bar{Q}^{k}\right], k \in \mathcal{S}_{Q} .
$$

\subsection{Constant reference voltage}

From the expression of losses given in section 2.3, it appears that the difference of voltages along the network is a source of energy losses. Therefore it is interesting to consider the case when $V^{D}$ is constant over $\mathcal{S}_{D}$. We may assume without loss of generality that $V^{D}=1$. Then $\hat{V}^{0}$ is constant and equal to 1 over $\mathcal{S}$. The expression of the first-order expansion simplifies to

$$
\left\{\begin{aligned}
(d \hat{\omega}) a+\sum_{\ell \neq k} y_{k \ell}\left(d \theta_{k}-d \theta_{\ell}\right) & =\varepsilon P_{k}-\sum_{\ell \neq k} g_{k \ell}, \quad k \in \mathcal{S}, \\
a^{t} d \theta & =0,
\end{aligned}\right.
$$

and

$$
\begin{cases}\sum_{\ell \neq k} y_{k \ell}\left(d \hat{V}_{k}-d \hat{V}_{\ell}\right) & =\varepsilon Q_{k}+\sum_{\ell \neq k} h_{k \ell}, \quad k \in \mathcal{S}_{Q}, \\ d \hat{V} & =0 \text { on } \mathcal{S}_{V}\end{cases}
$$

Note that $\eta$ is absent from these equations: it has no influence on the first-order term of the expansion of the solution. The expression of $d \hat{\omega}$ is

$$
d \hat{\omega}=\varepsilon \sum_{k \in \mathcal{S}} P_{k}-2 \sum_{k>\ell} g_{k \ell} .
$$


Therefore $d \hat{\omega}=0$ whenever $\sum_{k \in \mathcal{S}} P_{k}=\frac{2}{\varepsilon} \sum_{k>\ell} g_{k \ell}$, while the loss on line $k \ell$ satisfies by $(28)$

$$
\pi_{k \ell}=\frac{2 g_{k \ell}}{\varepsilon}\left(1+d \hat{V}_{k}+d \hat{V}_{\ell}\right)+\frac{1}{\varepsilon} O\left(\|\alpha\|^{3}\right)
$$

Remark In the particular case $d \hat{V}=0$, which is achieved by setting

$$
Q_{k}=-\frac{1}{\varepsilon} \sum_{\ell \neq k} h_{k \ell}, \quad k \in \mathcal{S}_{Q}
$$

then the voltage and the loss on line $k \ell$ satisfies

$$
V^{\varepsilon}=\frac{1}{\sqrt{\varepsilon}}+O\left(\frac{\|\alpha\|^{2}}{\sqrt{\varepsilon}}\right), \quad \pi_{k \ell}=\frac{2 g_{k \ell}}{\varepsilon}+\frac{1}{\varepsilon} O\left(\|\alpha\|^{3}\right) .
$$

\subsection{Active transit formulation}

Introducing the active transits in (15)-(18), in the same way we did in section 2 (i.e., changing $P_{k}, Q_{k}$ in $\varepsilon P_{k}, \varepsilon Q_{k}$ in the active transit formulation of section 2), we obtain the active transit formulation of the very high voltage approximation

$$
\begin{aligned}
\hat{T}_{k \ell}-\hat{V}_{k}^{2}\left(y_{k \ell} \sin \eta_{k \ell}+g_{k \ell}\right)-y_{k \ell} \hat{V}_{k} \hat{V}_{\ell} \sin \left(\theta_{k}-\theta_{\ell}-\eta_{k \ell}\right) & =0, k, \ell \in \mathcal{S}, \\
\hat{\omega} a+\sum_{\ell \neq k} \hat{T}_{k \ell} & =\varepsilon \hat{P}_{k}, k \in \mathcal{S}, \\
a^{t} \theta & =0, \\
\sum_{\ell \neq k} \hat{V}_{k}\left(y_{k \ell} \cos \eta_{k \ell}-h_{k \ell}\right)-y_{k \ell} \hat{V}_{\ell} \sqrt{1-\frac{\left(\hat{T}_{k \ell}-\hat{V}_{k}^{2}\left(y_{k \ell} \sin \eta_{k \ell}+g_{k \ell}\right)\right)^{2}}{\left(y_{k \ell} \hat{V}_{k} \hat{V}_{\ell}\right)^{2}}} & =\varepsilon \frac{\hat{Q}^{k}}{\hat{V}_{k}}, k \in \mathcal{S}_{Q}, \\
V & =V^{D} \text { on } \mathcal{S}_{V},
\end{aligned}
$$

where $\hat{V}:=\sqrt{\varepsilon} V$ and $\hat{\omega}:=\varepsilon \omega$ as before, and $\hat{T}_{k \ell}:=\varepsilon T_{k \ell}$. The associated limit system is

$$
\begin{aligned}
\hat{T}_{k \ell}-y_{k \ell} \hat{V}_{k} \hat{V}_{\ell} \sin \left(\theta_{k}-\theta_{\ell}\right) & =0, \quad k, \ell \in \mathcal{S}, \\
\hat{\omega} a+\sum_{\ell \neq k} \hat{T}_{k \ell} & =0, \quad k \in \mathcal{S}, \\
a^{t} \theta & =0, \\
\sum_{\ell \neq k} y_{k}\left[\hat{V}_{k}-\hat{V}_{\ell} \sqrt{1-\left(\frac{\hat{T}_{k \ell}}{y_{k \ell} \hat{V}_{k} \hat{V}_{\ell}}\right)^{2}}\right] & =0, \quad k \in \mathcal{S}_{Q}, \\
\hat{V} & =V^{D} \text { on } \mathcal{S}_{V} .
\end{aligned}
$$

$\mathrm{RR} \mathrm{n}^{\circ} 2544$ 
Lemma 2 The limit system has a unique solution in E, namely

$$
\hat{\omega}^{0}=0, \theta^{0}=0, \hat{T}^{0}=0, \hat{V}^{0},
$$

where $\hat{V}^{0}$ is given by Lemma 3.1. This solution is regular, and the active and reactive parts of the associated Jacobian are decoupled.

Proof Substituting the expression of $\hat{T}$ given by the first equation into the second and fifth equation we get the limit system (19)-(22). It follows that necessarily $\hat{\omega}^{0}=0, \theta^{0}=0$ and $\hat{V}^{0}$ is the same as the one computed in lemma 3.1. Finally the first equation gives $\hat{T}^{0}=0$.

The linearized limit equation with an arbitrary r.h.s. $\left(d t, d P, s, d Q, d V^{D}\right)$ is

$$
\begin{aligned}
d \hat{T}_{k \ell}-y_{k \ell} \hat{V}_{k} \hat{V}_{\ell}\left(d \theta_{k}-\theta_{\ell}\right) & =d t_{k \ell}, k \ell \in \mathcal{S}, \\
(d \hat{\omega}) a+\sum_{\ell \neq k} \hat{T}_{k \ell} & =d P_{k}, k \in \mathcal{S}, \\
a^{t} d \theta & =s \\
\sum_{\ell \neq k} y_{k \ell}\left(d V_{k}-d V_{\ell}\right) & =d Q, k \in \mathcal{S}_{Q}, \\
d \hat{V} & =d \hat{V}^{D} \text { on } \mathcal{S}_{V} .
\end{aligned}
$$

The decoupling property is obvious. We check that the Jacobian is invertible. The reactive part we obtain is identical to the one for the standard formulation, and is therefore invertible. Let us deal with the active part. Again, substituting the expression of $d \hat{T}_{k \ell}$ given by the first equation we obtain a linear system of the same nature as the linearization of (15)-(18) which was shown to be well posed. The unique value of $d \hat{T}$ being given by the fist equation, the linearized system has a unique solution.

\section{Algorithmic consequences}

\subsection{General framework}

We consider the possibility of solving the power flow equations using a Newton type method. We recall that Newton's method for solving

$$
F(x)=0,
$$

where $F$ is a $C^{1}$ mapping $\mathbb{R}^{n} \rightarrow \mathbb{R}^{n}$ (with Lipschitz continuous Jacobian), computes a sequence $\left\{x^{k}\right\}$ that, given $x^{k}$, gets $x^{k+1}$ out of the linear equation

$$
F\left(x^{k}\right)+F^{\prime}\left(x^{k}\right)\left(x^{k+1}-x^{k}\right)=0 .
$$


If $\bar{x}$ is a regular solution of (30), and $x^{0}$ is close enough to $\bar{x}$, then $x^{k}$ is well defined and $x^{k} \rightarrow \bar{x}$ quadratically (e.g. [11]). If no point close enough to the solution is known, then one has to use some device for "globalizing" the method, such as to use the gradient direction for computing a descent step on the associated least-square criterion $\|F(x)\|^{2}$, as suggested by Cauchy [8]. In this paper we confine ourself to the local study.

Sometimes, it is worth approximating the Jacobian $F^{\prime}\left(x^{k}\right)$ by a matrix $M^{k}$. In other words, the resulting Newton type algorithm computes a sequence $x^{k}$ satisfying

$$
F\left(x^{k}\right)+M^{k}\left(x^{k+1}-x^{k}\right)=0 .
$$

This is useful if the resulting linear system is easy to solve, e.g. if $M^{k}$ is block-diagonal. If $\bar{x}$ is a regular solution of (30), it is easily proved that if $x^{0}$ is closed to $\bar{x}$ and if $\left\{M^{k}\right\}$ remains close to $F^{\prime}(\bar{x})$, then $x^{k} \rightarrow \bar{x}$ at a linear rate (see e.g. [11]).

The system of equation we have dealt with until now is decoupled whenever $\alpha=0$. Also, the solution of the limit system is easily computed. Therefore, a natural idea is to solve this system of equations as follows: take as a starting point the solution of the limit system, and make a block-diagonal approximation of the Jacobian at each iteration.

\subsection{Application to the power flow problem}

Because the Jacobian of the limit system (for $\alpha=0$ ) is decoupled, we consider the Newton type algorithm for solving (15)-(18) in which the Jacobian is approximated by its "blockdiagonal" part. Let us set

$$
\begin{aligned}
p_{k} & :=\varepsilon P_{k}-\hat{\omega} a-\sum_{\ell \neq k} \hat{V}_{k}^{2}\left(y_{k \ell} \sin \eta_{k \ell}+g_{k \ell}\right)+y_{k \ell} \hat{V}_{k} \hat{V}_{\ell} \sin \left(\theta_{k}-\theta_{\ell}-\eta_{k \ell}\right), \\
q_{k} & :=\varepsilon \frac{Q_{k}}{\hat{V}_{k}}-\sum_{\ell \neq k} \hat{V}_{k}\left(y_{k \ell} \cos \eta_{k \ell}-h_{k \ell}\right)-y_{k \ell} \hat{V}_{\ell} \cos \left(\theta_{k}-\theta_{\ell}-\eta_{k \ell}\right) .
\end{aligned}
$$

The linear system to be solved at each iteration of the decoupled algorithm is as follows:

$$
\begin{aligned}
(d \omega) a+\sum_{\ell \neq k} y_{k \ell} \hat{V}_{k} \hat{V}_{\ell} \cos \left(\theta_{k}-\theta_{\ell}-\eta_{k \ell}\right)\left(d \theta_{k}-d \theta_{\ell}\right) & =p_{k}, \quad k \in \mathcal{S}, \\
a^{t} d \theta & =0,
\end{aligned}
$$

and

$$
\begin{array}{r}
\varepsilon \frac{Q_{k}}{\left(V_{k}\right)^{2}} d \hat{V}_{k}+\sum_{\ell \neq k}\left(y_{k \ell} \cos \eta_{k \ell}-h_{k \ell}\right) d \hat{V}_{k}-y_{k \ell} \cos \left(\theta_{k}-\theta_{\ell}-\eta_{k \ell}\right) d \hat{V}_{\ell} \\
=q_{k}, \quad k \in \mathcal{S}_{Q}, \\
d V=0 \text { on } \mathcal{S}_{V} .
\end{array}
$$

\section{Algorithm $S D$ (Standard Decoupling)}


0) Data $\left(\omega^{0}, \theta^{0}, \hat{V}^{0}\right)$ solution of the limit problem (19)-(22), $\varepsilon>0, j:=0$.

1) Set $\omega \leftarrow \omega^{j}, \theta \leftarrow \theta^{j}, \hat{V} \leftarrow \hat{V}^{j}$. Compute vectors $p$ and $q$ using (31).

2) Compute $(d \omega, d \theta, d \hat{V})$ solution of the above decoupled system.

3) $\left(\omega^{j+1}, \theta^{j+1}, \hat{V}^{j+1}\right) \leftarrow\left(\omega^{j}, \theta^{j}, \hat{V}^{h}\right)+(d \omega, d \theta, d \hat{V}) ; j \leftarrow j+1$; go to 1 .

\subsection{Active transit formulation}

The same type of decoupled algorithm may be stated for the active transit formulations. We just write the "linearization" of the reactive power equations, that we may write as follows

$$
\sum_{\ell \neq k} \hat{V}_{k}\left(y_{k \ell} \cos \eta_{k \ell}-h_{k \ell}\right)-\sqrt{\left(y_{k \ell} \hat{V}_{\ell}\right)^{2}-\left(\frac{T_{k \ell}}{\hat{V}_{k}}-y_{k \ell} \sin \eta_{k \ell}-g_{k \ell}\right)^{2}}=\frac{Q_{k}}{\hat{V}_{k}}, \quad k \in \mathcal{S}_{Q} .
$$

Set

$$
C_{k}:=\sqrt{\left(y_{k \ell} \hat{V}_{\ell}\right)^{2}-\left(\frac{T_{k \ell}}{\hat{V}_{k}}-y_{k \ell} \sin \eta_{k \ell}-g_{k \ell}\right)^{2}} .
$$

The associated "linearized" system with block diagonal decoupling is, writing only the reactive part:

$$
\frac{Q_{k}}{\left(\hat{V}_{k}\right)^{2}} d \hat{V}_{k}+\sum_{\ell \neq k}\left(y_{k \ell} \cos \eta_{k \ell}-h_{k \ell}\right) d \hat{V}_{k}-\frac{1}{C_{k}}\left[y_{k \ell}^{2} V_{\ell} d V_{\ell}+\left(\frac{T_{k \ell}}{\hat{V}_{k}}-y_{k \ell} \sin \eta_{k \ell}-g_{k \ell}\right) \frac{T_{k \ell}}{\left(\hat{V}_{k}\right)^{2}} d \hat{V}_{k}\right]=q_{k}
$$

\subsection{Analysis of convergence}

The following framework will be convenient for our study. Consider a family of problem

$$
F_{\varepsilon}(x)=0
$$

with $\bar{x}$ a regular solution of $F_{0}$ and $(\varepsilon, x) \rightarrow F_{\varepsilon}(x)$ an analytical mapping. Then, by the implicit function theorem, there exists locally (i.e. for $(x, \varepsilon)$ close to $(\bar{x}, 0)$ ) a unique solution $x^{\varepsilon}$, and $\varepsilon \rightarrow x^{\varepsilon}$ is itself analytical. We consider the following algorithm for computing $x^{\varepsilon}$ :

\section{Algorithm $G$ (Général)}

$0)$ Data $x^{0} \leftarrow \bar{x}, \varepsilon>0, j \leftarrow 0$.

1) Choose $M^{j}>0$ satisfying for some $c$ (not depending on $\varepsilon$ and $j$ )

$$
\left\|M^{j}-F_{\varepsilon}^{\prime}\left(x^{j}\right)\right\| \leq c\left(\varepsilon+\left\|x^{j}-\bar{x}\right\|\right) .
$$

2) Compute $x^{j+1}$ solution of

$$
F_{\varepsilon}\left(x^{j}\right)+M^{j}\left(x^{j+1}-x^{j}\right)=0 .
$$

3) $j:=j+1$; go to 1 . 
So we assume that the starting point is the solution of the limit system. Note that in our application the solution of the limit system is easily computed and relation (32) is satisfied. The following theorem shows that for $\varepsilon$ small enough the algorithm converges rapidly:

Theorem 2 Fix $c>0$. Then there exists $c^{\prime}>0$ such that if (32) holds then for small enough $\varepsilon$, the sequence $\left\{x^{k}\right\}$ is well-defined and converges towards $x^{\varepsilon}$, and

$$
\left\|x^{j+1}-x^{\varepsilon}\right\| \leq \varepsilon c^{\prime}\left\|x^{j}-x^{\varepsilon}\right\| .
$$

\section{Proof}

Let $L$ and $L^{\prime}$ be Lipschitz constants for $F_{\varepsilon}$ and $F_{\varepsilon}^{\prime}$ (w.r.t. $x$ ) whenever

$$
\varepsilon \leq \varepsilon_{1} \text { and }\|x-\bar{x}\| \leq \varepsilon_{1}
$$

with $\varepsilon_{1}>0$. Let $\varepsilon_{2}>0$ be such that $\left\|x^{\varepsilon}-\bar{x}\right\| \leq \varepsilon_{1}$ whenever $\varepsilon \leq \varepsilon_{2}$. If (34) is satisfied and $\varepsilon \leq \varepsilon_{2}$, then

$$
\begin{aligned}
F_{\varepsilon}(x) & =F_{\varepsilon}\left(x^{\varepsilon}\right)+\left[\int_{0}^{1} F_{\varepsilon}^{\prime}\left(x^{\varepsilon}+\sigma\left(x-x^{\varepsilon}\right)\right) d \sigma\right]\left(x-x^{\varepsilon}\right), \\
& =F_{\varepsilon}^{\prime}\left(x^{\varepsilon}\right)\left(x-x^{\varepsilon}\right)+\int_{0}^{1}\left[F_{\varepsilon}^{\prime}\left(x^{\varepsilon}+\sigma\left(x-x^{\varepsilon}\right)\right)-F_{\varepsilon}^{\prime}\left(x^{\varepsilon}\right)\right] d \sigma\left(x-x^{\varepsilon}\right) .
\end{aligned}
$$

We deduce that

$$
\left\|F_{\varepsilon}(x)-F_{\varepsilon}^{\prime}\left(x^{\varepsilon}\right)\left(x-x^{\varepsilon}\right)\right\| \leq \frac{L^{\prime}}{2}\left\|x-x^{\varepsilon}\right\|^{2} .
$$

The error after an exact Newton step is

$$
T_{\varepsilon}(x):=x-F_{\varepsilon}^{\prime}\left(x^{\varepsilon}\right)^{-1} F_{\varepsilon}(x)-x^{\varepsilon}=F_{\varepsilon}^{\prime}\left(x^{\varepsilon}\right)^{-1}\left[F_{\varepsilon}^{\prime}\left(x^{\varepsilon}\right)\left(x-x^{\varepsilon}\right)-F_{\varepsilon}(x)\right] .
$$

Therefore

$$
\left\|T_{\varepsilon}(x)\right\|=\left\|F_{\varepsilon}^{\prime}\left(x^{\varepsilon}\right)^{-1}\left[F_{\varepsilon}^{\prime}\left(x^{\varepsilon}\right)\left(x-x^{\varepsilon}\right)-F_{\varepsilon}(x)\right]\right\| \leq \frac{L^{\prime}}{2}\left\|F_{\varepsilon}^{\prime}\left(x^{\varepsilon}\right)^{-1}\right\|\left\|x-x^{\varepsilon}\right\|^{2} .
$$

Now if $M$ is an invertible matrix, we want to compute the error after the Newton type step

$$
T^{M}(x):=x-M^{-1} F(x)-x^{\varepsilon} .
$$

We have, reducing $\varepsilon$ if necessary

$$
\begin{aligned}
\left\|T^{M}(x)\right\| & =\left\|T_{\varepsilon}(x)+\left(F_{\varepsilon}^{\prime}\left(x^{\varepsilon}\right)^{-1}-M^{-1}\right) F(x)\right\|, \\
& \leq \frac{L^{\prime}}{2}\left\|F_{\varepsilon}^{\prime}\left(x^{\varepsilon}\right)^{-1}\right\|\left\|x-x^{\varepsilon}\right\|^{2}+\left\|M^{-1}-F_{\varepsilon}^{\prime}\left(x^{\varepsilon}\right)^{-1}\right\|\|F(x)\|, \\
& \leq L\left\|F_{0}^{\prime}(\bar{x})^{-1}\right\|\left\|x-x^{\varepsilon}\right\|^{2}+L\left\|M^{-1}-F_{\varepsilon}^{\prime}\left(x^{\varepsilon}\right)^{-1}\right\|\left\|x-x^{\varepsilon}\right\| .
\end{aligned}
$$

$\mathrm{RR} \mathrm{n}^{\circ} 2544$ 
Now if (34) is satisfied and

$$
\left\|M-F_{0}^{\prime}(\bar{x})\right\|<\varepsilon_{3}
$$

for a certain $\varepsilon_{3}>0$ small enough we have

$$
\begin{aligned}
\left\|M^{-1}-F_{\varepsilon}^{\prime}\left(x^{\varepsilon}\right)^{-1}\right\| & =\left\|F_{\varepsilon}^{\prime}\left(x^{\varepsilon}\right)^{-1}\left(F_{\varepsilon}^{\prime}\left(x^{\varepsilon}\right)-M\right) M^{-1}\right\|, \\
& \leq\left\|M^{-1}\right\|\left\|F_{\varepsilon}^{\prime}\left(x^{\varepsilon}\right)^{-1}\right\|\left\|M-F_{\varepsilon}^{\prime}\left(x^{\varepsilon}\right)\right\|, \\
& \leq 2\left\|F_{0}^{\prime}(\bar{x})^{-1}\right\|^{2}\left\|M-F_{\varepsilon}^{\prime}\left(x^{\varepsilon}\right)\right\|,
\end{aligned}
$$

and

$$
\begin{aligned}
\left\|M-F_{\varepsilon}^{\prime}\left(x^{\varepsilon}\right)\right\| & \leq\left\|M-F_{\varepsilon}^{\prime}(x)\right\|+\left\|F_{\varepsilon}^{\prime}(x)-F_{\varepsilon}^{\prime}\left(x^{\varepsilon}\right)\right\|, \\
& \leq\left\|M-F_{\varepsilon}^{\prime}(x)\right\|+L^{\prime}\left\|x-x^{\varepsilon}\right\| .
\end{aligned}
$$

Combining with the above relations, we get whenever (34) and (35) hold

$$
\left\|T^{M}(x)\right\| \leq \varepsilon c^{\prime}\left\|x-x^{\varepsilon}\right\|,
$$

where

$$
c^{\prime}:=\frac{L^{\prime}}{\varepsilon}\left\|F_{0}^{\prime}(\bar{x})^{-1}\right\|\left\|x-x^{\varepsilon}\right\|+\frac{2 L}{\varepsilon}\left\|F_{0}^{\prime}(\bar{x})^{-1}\right\|^{2}\left(\left\|M-F_{\varepsilon}^{\prime}(x)\right\|+L^{\prime}\left\|x-x^{\varepsilon}\right\|\right),
$$

i.e. $c^{\prime}=c^{\prime}(x, M, \varepsilon)$ satisfies for some $c_{1}>0$ and $c_{2}>0$

$$
c^{\prime}(x, M, \varepsilon) \leq \frac{c_{1}}{\varepsilon}\left\|x-x^{\varepsilon}\right\|+\frac{c_{2}}{\varepsilon}\left\|M-F_{\varepsilon}^{\prime}(x)\right\| .
$$

We now prove by induction that the conclusion of the theorem holds as well as

$$
\left\|x^{j+1}-x^{\varepsilon}\right\| \leq \frac{1}{2}\left\|x^{j}-x^{\varepsilon}\right\| .
$$

Assume that this holds until iteration $j$. Let $c_{3}$ be such that $\left\|x^{\varepsilon}-\bar{x}\right\| \leq \varepsilon c_{3}$ for $\varepsilon$ small enough. Then

$$
\left\|x^{j}-\bar{x}\right\| \leq\left\|x^{j}-x^{\varepsilon}\right\|+\left\|x^{\varepsilon}-\bar{x}\right\| \leq 2\left\|x^{\varepsilon}-\bar{x}\right\| \leq 2 \varepsilon c_{3},
$$

hence with (32)

$$
\left\|M^{j}-F_{\varepsilon}^{\prime}\left(x^{j}\right)\right\| \leq c\left(\varepsilon+2 \varepsilon c_{3}\right)=\varepsilon c\left(1+2 c_{3}\right) .
$$

It follows that when $\varepsilon$ is small enough, we have $\varepsilon c\left(1+2 c_{3}\right)<\varepsilon_{2}$, whence (35) holds, (34) also holds, by (36), we have $c^{\prime}(x, M, \varepsilon) \leq c_{1}^{\prime}$, where

$$
c_{1}^{\prime}:=c_{1} c_{3}+c_{2} c\left(1+2 c_{3}\right) \text {. }
$$

It follows that when $\varepsilon \leq\left(2 c_{1}^{\prime}\right)^{-1}$, then (37) and (33) holds. The conclusion follows. 


\section{Network with purely imaginary impedances}

Until here we have dealt with the full system of power flow equations. However it may be useful to concentrate on the dependence on parameter $\varepsilon$. For this purpose we set the other small parameters $(\eta, g, h)$ to 0 . As we will see, some very specific properties are obtained in this case.

\subsection{The potential formulation}

When $(\eta, g, h)$ is set to 0 , the expression of the power flow equations, in the framework of the high voltage approximation, is (see (15)-(18)):

$$
\begin{aligned}
\hat{\omega} a+\sum_{\ell \neq k} y_{k \ell} \hat{V}_{k} \hat{V}_{\ell} \sin \left(\theta_{k}-\theta_{\ell}\right) & =\varepsilon P_{k}, k \in \mathcal{S}, \\
a^{t} \theta & =0, \\
\sum_{\ell \neq k} y_{k \ell}\left[\hat{V}_{k}-\hat{V}_{\ell} \cos \left(\theta_{k}-\theta_{\ell}\right)\right] & =\varepsilon \frac{Q_{k}}{\hat{V}_{k}}, k \in \mathcal{S}_{Q}, \\
\hat{V} & =V^{D} \text { on } \mathcal{S}_{V} .
\end{aligned}
$$

We say that an equation $F(x, \lambda)=0$, where $(x, \lambda) \in \mathbb{R}^{n} \times \mathbb{R}^{p}$, is the equation of a critical point of a potential $f: \mathbb{R}^{n} \rightarrow \mathbb{R}$ over a manifold $\left\{x \in \mathbb{R}^{n} ; g(x)=0\right\}$, where $g: \mathbb{R}^{n} \rightarrow \mathbb{R}^{p}$, if

$$
F(x, \lambda)=\left(\begin{array}{c}
\nabla f(x)+g^{\prime}(x)^{T} \lambda \\
g(x)
\end{array}\right)
$$

and we call $\lambda$ a Lagrange multiplier associated with the constraint $g(x)=0$. We observe that this framework fits to system (38)-(41):

Proposition 1 System (38)-(41) is nothing but the equation of a critical point of the potential below:

$$
\begin{aligned}
\mathcal{P}_{\varepsilon}(\theta, \hat{V}):= & \frac{1}{2} \sum_{k>\ell} y_{k \ell}\left(\hat{V}_{k}^{2}+\hat{V}_{\ell}^{2}\right)-\sum_{k>\ell} y_{k \ell} \hat{V}_{k} \hat{V}_{\ell} \cos \left(\theta_{k}-\theta_{\ell}\right) \\
& -\varepsilon \sum_{k \in \mathcal{S}} \theta_{k} P_{k}-\varepsilon \sum_{k \in \mathcal{S}_{Q}} Q_{k} \log \hat{V}_{k}
\end{aligned}
$$

over the set

$$
\left\{(\theta, \hat{V}) ; a^{t} \theta=0 ; \hat{V}=V^{D} \text { on } \mathcal{S}_{V}\right\},
$$

the parameter $\hat{\omega}$ being interpretated as a Lagrange multiplier associated with the first constraint.

$\operatorname{RR} n^{\circ} 2544$ 
We note that an equivalent expression of the potential is

$$
\begin{gathered}
\mathcal{P}_{\varepsilon}(\theta, \hat{V}):=\frac{1}{2} \sum_{k>\ell} y_{k \ell}\left[\left(\hat{V}_{k}-\hat{V}_{\ell}\right)^{2}+\hat{V}_{k} \hat{V}_{\ell}\left(1-\cos \left(\theta_{k}-\theta_{\ell}\right)\right)\right] \\
-\varepsilon \sum_{k \in \mathcal{S}} \theta_{k} P_{k}-\varepsilon \sum_{k \in \mathcal{S}_{Q}} Q_{k} \log \hat{V}_{k} .
\end{gathered}
$$

\subsection{Study of the potential}

The theorem below shows that when $\varepsilon$ is close to 0 , the problem of minimizing the potential is locally equivalent to the one of solving the power flow equations.

Theorem 3 Let $\beta$ satisfy (23). When $\varepsilon$ is close enough to 0 , the power flow problem has a unique solution in $E_{\beta}$, which is a strict local minimum of the potential.

Proof Proceeding as in the proof of Theorem 1 , we can check that (i) if $\left(\hat{\omega}^{\varepsilon}, \theta^{\varepsilon}, \hat{V}^{\varepsilon}\right)$ is a solution of (38)-(41) in $E_{\beta}$ and $\varepsilon \downarrow 0$, then $\left(\hat{\omega}^{\varepsilon}, \theta^{\varepsilon}, \hat{V}^{\varepsilon}\right) \rightarrow\left(\hat{\omega}^{0}=0, \theta^{0}=0, \hat{V}^{0}\right)$ solution of the limit system (19)-(22) and (ii) the Jacobian of this limit system is invertible and therefore we may apply the implicit function theorem in the vicinity of the limit solution. It follows that for $\varepsilon$ small enough the power flow problem has a unique solution.

Let us now come back to the abstract framework of critical points, assuming the gradients of constraints to be linearly independent. From optimization theory, we know that if the second-order necessary condition for optimality holds, i.e. the Hessian of the cost is nonnegative along any direction that is tangent to the feasible set, then the Jacobian of the optimality system is invertible iff the second order sufficient optimality conditions holds, i.e. the Hessian of the cost is actually positive definite along any tangent direction (see e.g. [6]). This is a sufficient condition for optimality that remains stable for sufficiently small $\varepsilon$. The Jacobian being invertible in our application, it suffices to check the second-order necessary condition for the limit problem. Note that by (43) the limit potential $\mathcal{P}_{0}$ reduces to

$$
\mathcal{P}_{0}(\theta, \hat{V})=\frac{1}{2} \sum_{k>\ell} y_{k \ell}\left[\left(\hat{V}_{k}-\hat{V}_{\ell}\right)^{2}+2 \hat{V}_{k} \hat{V}_{\ell}\left(1-\cos \left(\theta_{k}-\theta_{\ell}\right)\right)\right]
$$

It suffices to check that the Hessian of each term of the sum is nonnegative. Because $\theta \rightarrow$ $\theta_{k}-\theta_{\ell}$ is a linear mapping, it suffices in fact to check that the mapping

$$
\left(\delta, V_{1}, V_{2}\right) \rightarrow \frac{1}{2}\left(V_{k}-V_{\ell}\right)^{2}+V_{k} V_{\ell}(1-\cos \delta)
$$

has a nonnegative Hessian at all point satisfying $\delta=0$. We compute the gradient

$$
g=\left[V_{1} V_{2} \sin \delta, V_{1}-V_{2} \cos \delta, V_{2}-V_{1} \cos \delta\right]^{t},
$$


and the Hessian

$$
H=\left[\begin{array}{ccc}
V_{1} V_{2} \cos \delta & V_{2} \sin \delta & V_{1} \sin \delta \\
r V_{2} \sin \delta & 1 & -\cos \delta \\
r V_{1} \sin \delta & -\cos \delta & 1
\end{array}\right]
$$

whose determinant is

$$
-\left(V_{1} V_{2} \cos \delta+V_{2}^{2}+V_{1}^{2}\right) \sin ^{2} \delta \leq 0 .
$$

As trace $(H)>0$ (remember that $\cos \delta>0$ as $\theta \in \Theta$ ), there is a unique negative eigenvalue iff $\delta \neq 0$. However, for the limiting solution, we obtain

$$
h=\left[\begin{array}{ccc}
V_{1} V_{2} & 0 & 0 \\
r 0 & 1 & -1 \\
r 0 & -1 & 1
\end{array}\right] \text { with eigenvalues } V_{1} V_{2}, 1,0,
$$

so that $H$ is nonnegative, as was to be proved.

Remark It is easily checked that $\theta \rightarrow \pi(\theta, V)$ is convex over $E$, using the fact that $\delta \rightarrow-\cos \delta$ is convex over $[-\pi / 2, \pi / 2]$, as the second derivative is $\cos \delta \geq 0$.

\subsection{Formulation using active transits}

Setting $(\eta, g, h)$ to 0 in the active transit formulation (section 3.5), we get the following system

$$
\begin{aligned}
\hat{T}_{k \ell}-y_{k \ell} \hat{V}_{k} \hat{V}_{\ell} \sin \left(\theta_{k}-\theta_{\ell}\right) & =0, \quad k, \ell \in \mathcal{S}, \\
\omega a+\sum_{\ell \neq k} T_{k \ell} & =\varepsilon P_{k}, \quad k \in \mathcal{S}, \\
a^{t} \theta & =0 \\
\sum_{\ell \neq k} y_{k \ell} \hat{V}_{k}-y_{k \ell} \sqrt{\hat{V}_{\ell}^{2}-\left(\frac{\hat{T}_{k \ell}}{y_{k \ell} \hat{V}_{k}}\right)^{2}} & =\varepsilon \frac{Q_{k}}{V_{k}}, \quad k \in \mathcal{S}_{Q} \\
\hat{V} & =V^{D} \text { on } \mathcal{S}_{V} .
\end{aligned}
$$

We will study separately the active and reactive part of this system (i.e. the three first and two last equations), obtaining for each of them a variational formulation.

Study of the active part The sine function is one-to-one from $[-\pi / 2, \pi / 2]$ onto $[-1,1]$. Consequently, the reciprocal function arcsin is well defined and one-to-one from $[-1,1]$ onto $[-\pi / 2, \pi / 2]$. Let us denote as $\int$ as the primitive of arcsin, over $[-1,1]$. Chosing the integration constant so as to obtain $\int \operatorname{as}(0)=0$, we obtain

$$
\int \operatorname{as}(x)=x \arcsin x+\sqrt{1-x^{2}}-1 .
$$

As arcsin is strictly monotonously increasing, $\int \operatorname{as}(x)$ is a strictly convex function.

$\mathrm{RR} \mathrm{n}^{\circ} 2544$ 
Proposition 2 The active power equations are equivalent to say that $T_{k \ell}=0$ whenever $y_{k \ell}=0, T_{k \ell}:-T_{\ell k}$ if $\ell>k$, and $(\omega, T)$ is a global solution of the convex problem

$$
\begin{array}{r}
\operatorname{Min}_{(\omega, T) \in \mathbb{R} \times \mathcal{T}} \Xi(T):=\sum_{\substack{k>\ell \\
y_{k \ell \neq 0}}} y_{k \ell} \hat{V}_{k} \hat{V}_{\ell} \int \operatorname{as}\left(\frac{T_{k \ell}}{y_{k \ell} \hat{V}_{k} \hat{V}_{\ell}}\right), \\
\text { s.t. } \omega a+\sum_{k>\ell} T_{k \ell}-\sum_{\ell>k} T_{k \ell}=\varepsilon P_{k}, \forall k \in \mathcal{S},
\end{array}
$$

and the set of feasible active transits is

$$
\mathcal{T}=\left\{T_{k \ell} \in \mathbb{R} ; k>\ell \text { and } y_{k \ell} \neq 0\right\} .
$$

The solution of this problem (if any) is unique and $\theta$ (satisfying (44) and (46)) may be interpreted as a Lagrange multiplier associated with the above problem.

Proof Let us introduce the Lagrangian function

$$
\mathcal{L}(\omega, T, \theta):=\Xi(T)-\sum_{k \in \mathcal{S}} \theta_{k}\left[w a_{k}+\sum_{k>\ell} T_{k \ell}-\sum_{\ell>k} T_{\ell k}-\varepsilon P_{k}\right] .
$$

Problem (50) has a convex cost and linear constraints. Consequently its solution are characterized by the optimality system (where $\theta$ is the Lagrange multiplier associated with the constraint)

$$
\begin{cases}\arcsin \left(\frac{T_{k \ell}}{y_{k \ell} \hat{V}_{k} \hat{V}_{\ell}}\right) & =\theta_{k}-\theta_{\ell}, \forall k, \ell \in \mathcal{S} ; k>\ell, y_{k \ell}>0, \\ \omega a+\sum_{k>\ell} T_{k \ell}-\sum_{\ell>k} T_{k \ell} & =\varepsilon P_{k}, k \in \mathcal{S}, \\ a^{t} \theta & =0 .\end{cases}
$$

Setting $T_{\ell k}=-T_{k \ell}$ when $\ell>k$, and noticing that (44) implies that $T_{k \ell}=0$ if $y_{k \ell}=0$, we see that (44) - (46) is equivalent to (51) and the requirement that $T_{k \ell}=0$ whenever $y_{k \ell}=0$.

Now $\Xi$ is a sum of strictly convex functions of components of $T_{k \ell}, k>\ell, y_{k \ell} \neq 0$. It follows that if $\left(\omega^{1}, T^{1}\right)$ and $\left(\omega^{2}, T^{2}\right)$ are solution of $(50)$, then $T^{1}=T^{2}$. From the constraint of $(50)$ we deduce then that $\omega^{1}=\omega^{2}$.

We observe that (50)-(51) may be interpreted as a nonlinear direct current problem, in which $T$ represents the currents and $\theta$ the voltage. The nonlinearity of the resistance is reflected in the non quadraticity of the potential $\Xi$. To the "current" formulation (50) we may associate a dual "voltage" formulation.

The dual cost is computed by minimizing the Lagrangian function with respect to the primal variables $(\omega, T)$. Minimizing w.r.t. $\omega$ amounts to put $(46)$ as a dual constraints. 
Minimizing w.r.t. $T$ we recover (44). Set $\chi:=T_{k \ell} / y_{k \ell} \hat{V}_{k} \hat{V}_{\ell}$. Then $\chi=\sin \left(\theta_{k}-\theta_{\ell}\right)$. Using (49) we get

$$
\begin{aligned}
\int \operatorname{as} \chi & =\chi\left(\theta_{k}-\theta_{\ell}\right)+\sqrt{1-\sin \left(\theta_{k}-\theta_{\ell}\right)^{2}}-1 \\
& =\left(\theta_{k}-\theta_{\ell}\right) \sin \left(\theta_{k}-\theta_{\ell}\right)+\cos \left(\theta_{k}-\theta_{\ell}\right)-1 .
\end{aligned}
$$

Substituting this expression in $\Xi(T)$, and simplifying the expression of the resulting dual cost, we obtain the dual problem

$$
\operatorname{Max}_{\theta} \sum_{\substack{k>\ell \\ y_{k \ell}>0}} y_{k \ell} \hat{V}_{k} \hat{V}_{\ell}\left[\cos \left(\theta_{k}-\theta_{\ell}\right)-1\right]+\varepsilon \sum_{k \in \mathcal{S}} P_{k} \theta_{k} ; a^{t} \theta=0 .
$$

Note that maximizing this potential with respect to $\theta$ is equivalent to the minimization of $\mathcal{P}_{\varepsilon}(\theta, \hat{V})$ w.r.t. $\theta$.

Study of the reactive part For the limit system, the reactive power equations are, up to the first order, insensitive to a variation of $T$. Therefore, it is important to understand these equations as function of $V$, for a given $T$. We will give the expression of an associated potential. For this we need to consider the secant function $\sec x:=1 / \cos x$, which is monotonously strictly increasing and one-to-one: $[0, \pi / 2[\rightarrow[1, \infty[$. At a point $x \in[0, \pi / 2[$, set $y:=\sec x$. Then

$$
(\sec x)^{\prime}=\frac{\sin x}{\cos ^{2} x}=y^{2} \sqrt{1-1 / y^{2}} .
$$

Therefore, the inverse function $\operatorname{arcsec} x:[1, \infty[\rightarrow[0, \pi / 2$ [is strictly increasing and satisfies

$$
(\operatorname{arcsec} y)^{\prime}=\frac{1}{y^{2} \sqrt{1-1 / y^{2}}}
$$

and therefore

$$
\left[y\left(\sqrt{1-1 / y^{2}}-\frac{1}{y} \operatorname{arcsec} y\right)\right]^{\prime}=\left(y \sqrt{1-1 / y^{2}}-\operatorname{arcsec} y\right)^{\prime}=\sqrt{1-1 / y^{2}} .
$$

Finally we define the potential

$$
\begin{aligned}
& \tilde{\mathcal{P}}_{\varepsilon}(\hat{V}):=\frac{1}{2} \sum_{k>\ell} y_{k \ell}\left(\hat{V}_{k}^{2}+\hat{V}_{\ell}^{2}\right)-\varepsilon \sum_{k \in \mathcal{S}_{Q}} Q_{k} \log \hat{V}_{k} \\
& \quad-\sum_{\substack{k>\ell \\
T_{k \ell \neq 0}}} y_{k \ell} \hat{V}_{k} \hat{V}_{\ell}\left[\sqrt{1-\left(\frac{\hat{T}_{k \ell}}{y_{k \ell} \hat{V}_{k} \hat{V}_{\ell}}\right)^{2}}-\frac{\left|\hat{T}_{k \ell}\right|}{y_{k \ell} \hat{V}_{k} \hat{V}_{\ell}} \operatorname{arcsec}\left(y_{k \ell} \frac{\hat{V}_{k} \hat{V}_{\ell}}{\left|\hat{T}_{k \ell}\right|}\right)\right] .
\end{aligned}
$$

A more compact notation is obtained by using

$$
S_{k \ell}:=\hat{T}_{k \ell} / y_{k \ell} \hat{V}_{k} \hat{V}_{\ell}, \quad C_{k \ell}:=\sqrt{1-S_{k \ell}^{2}}
$$

$\mathrm{RR} \mathrm{n}^{\circ} 2544$ 
which are equal to the sine and cosine of difference of phases if the active equations are satisfied. We get

$$
\begin{aligned}
\tilde{\mathcal{P}}_{\varepsilon}(V) & :=\frac{1}{2} \sum_{k \neq \ell} y_{k \ell}\left(\hat{V}_{k}^{2}+\hat{V}_{\ell}^{2}\right) \\
& -\sum_{\substack{k>\ell \\
T_{k \ell} \neq 0}} y_{k \ell} \hat{V}_{k} \hat{V}_{\ell}\left[C_{k \ell}-\left|S_{k \ell}\right| \operatorname{arcsec}\left(\frac{1}{\left|S_{k \ell}\right|}\right)\right]-\varepsilon \sum_{k \in \mathcal{S}_{Q}} Q_{k} \log \hat{V}_{k} .
\end{aligned}
$$

Because arcsec if defined on $[1, \infty]$ and differentiable over $] 1, \infty[$, we take as domain of definition of $\tilde{\mathcal{P}}_{\varepsilon}$ the open convex set

$$
D\left(\tilde{\mathcal{P}}_{\varepsilon}\right):=\left\{\hat{V} \in \mathbb{R}_{++}^{n} ; \hat{V}_{k} \hat{V}_{\ell}>\left|\hat{T}_{k \ell}\right| / y_{k \ell}, \forall k, \ell \in \mathcal{S} ; \hat{T}_{k \ell} \neq 0\right\} .
$$

Proposition 3 (i) The reactive power equations (47) are nothing but the equations of critical points for $\tilde{\mathcal{P}}_{\varepsilon}$ over $D\left(\tilde{\mathcal{P}}_{\varepsilon}\right)$.

(ii) Let $\beta$ satisfy (23). When $(\varepsilon, \hat{T})$ is close enough to 0 , then equation (47) has a unique solution over $V_{\beta}$ which is a local minimum of $\tilde{\mathcal{P}}_{\varepsilon}$.

Proof Point (i) is obtained by computing the gradient of $\tilde{\mathcal{P}}_{\varepsilon}$, using (52). Now let us prove (ii). We first prove that when $(\varepsilon, \hat{T}) \rightarrow 0$, then the set of solutions of $(47)$ is bounded. Let us write (47) as

$$
\begin{cases}\sum_{\ell=k} y_{k \ell}\left(\hat{V}_{k}-\hat{V}_{\ell}\right)=\varepsilon Q_{k} / \hat{V}_{k}-y_{k \ell} \sum_{\ell \neq k} \hat{V}_{\ell}\left(1-C_{k \ell}\right) & \text { on } \mathcal{S}_{Q}, \\ \hat{V} \neq V^{D} & \text { on } \mathcal{S}_{V},\end{cases}
$$

where $C_{k \ell} \rightarrow 1$ as $(\varepsilon, \hat{T}) \rightarrow 0$. Therefore, as $\min V_{k} \geq \beta>0$ :

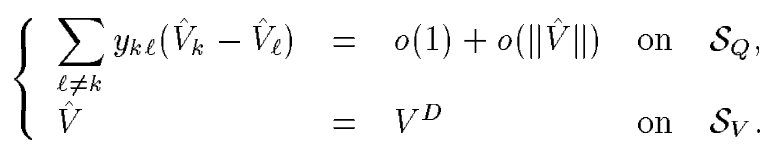

Because $\mathcal{S}_{V} \neq \emptyset$ and the network is connected, the linear operator in the left hand side is invertible. We deduce that

$$
\hat{V}=O\left(\left\|V^{D}\right\|\right)+o(1)+o(\|\hat{V}\|)
$$

which gives a uniform bound on $\hat{V}$.

The choice of $V_{\beta}$ preventing any component of $\hat{V}$ to go to 0 , we may then pass to the limit in (47). We deduce that any solution of (47) in $V_{\beta}$ converges to $\hat{V}^{0}$ as $(\varepsilon, \hat{T}) \rightarrow 0$.

Now for $(\varepsilon, \hat{T})=0$, the unique solution $\hat{V}^{0}$ is regular: its Jacobian equal to the Hessian of $\tilde{\mathcal{P}}_{\varepsilon}$ is invertible. Therefore, in order to show that this Hessian is positive definite, it suffices to prove that it is semidefinite positive. Then by continuity, for $(\varepsilon, \hat{T}, \hat{V})$ close enough to 
$\left(0,0, \hat{V}^{0}\right)$ this Jacobian will remain positive definite at the solution, which will prove that the solution is a local minimum of $\tilde{\mathcal{P}}_{\varepsilon}$. So we compute the Hessian of $\tilde{\mathcal{P}}_{\varepsilon}$ for $\varepsilon=0$. It suffices to prove that each term of the sum over the effective lines has a nonnegative Hessian. That is we study (dividing by $y_{k \ell}$ ):

$$
\left(V_{k}, V_{\ell}\right) \rightarrow \varphi\left(V_{k}, V_{\ell}\right):=\frac{1}{2}\left(\hat{V}_{k}^{2}+\hat{V}_{\ell}^{2}\right)-\sqrt{\hat{V}_{k}^{2} \hat{V}_{\ell}^{2}-\sigma_{k \ell}^{2}}+\sigma_{k \ell} \operatorname{arcsec} \frac{\hat{V}_{k} \hat{V}_{\ell}}{\sigma_{k \ell}},
$$

where we have set $\sigma_{k \ell}:=\left|T_{k \ell}\right| / y_{k \ell}$. Computing the gradient, we recover of course the corresponding term in (47):

$$
\frac{\partial \varphi}{\partial V_{k}}=V_{k}-\sqrt{\hat{V}_{\ell}^{2}-\left(\frac{\sigma_{k \ell}}{\hat{V}_{k}}\right)^{2}}
$$

We obtain

$$
\nabla^{2} \varphi\left(\hat{V}_{k}, \hat{V}_{\ell}\right)=\left(\begin{array}{cc}
1-\frac{\hat{V}_{\ell}}{\hat{V}_{k}} \frac{S_{k \ell}^{2}}{C_{k \ell}} & -1 / C_{k \ell} \\
-1 / C_{k \ell} & 1-\frac{\hat{V}_{k}}{\hat{V}_{\ell}} \frac{S_{k \ell}^{2}}{C_{k \ell}}
\end{array}\right),
$$

where $S_{k \ell}$ and $C_{k \ell}$ are defined by (54). When $\sigma \rightarrow 0$ we end up with

$$
\nabla^{2} \varphi\left(\hat{V}_{k}, \hat{V}_{\ell}\right)=\left(\begin{array}{cc}
1 & -1 \\
-1 & 1
\end{array}\right)
$$

whose eigenvalues are 0 and 1 , and therefore nonnegative, as was to be proved.

\section{References}

[1] J.P. Aubin, P.A. Raviart, On the resolution of equations arising in load flow problems. Proc. IEEE Power Industry Computer Applications Conf., 1965, pp. 119-132.

[2] G. Blanchon, J.C. Dodu. Introduction de la technique CRIC dans les modèles de planification de l'énergie réactive. Note EDF, 1993.

[3] G. Blanchon, J.F. Bonnans, J.C. Dodu, Optimisation de réseaux électriques de grande taille. Lect. Notes in Information and Control Sciences 144, Springer Verlag, Berlin, 1990, pp. 423-431.

[4] J.F. Bonnans: Rapport de Contrat EDF-INRIA, 1994.

[5] J.F. Bonnans: Mathematical study of very high voltage power networks I: The optimal DC power flow problem. Rapport de Recherche INRIA nº 2408, November 1994.

[6] J.F. Bonnans, A. Sulem: Pseudopower expansion of solutions of generalized equations and constrainted optimization problems. Mathematical Programming, to appear. 
[7] C. Carpentier, CRIC : a new active-reactive decoupling process in load flows, optimal power flows and system control, in Proc. IFAC Conf. Power Systems and Power Plant Control, Beijing, 1986, pp. 65-70.

[8] A. Cauchy, Méthode générale pour la résolution des systèmes d'équations simultanées. Comptes-Rendus Acad. Sciences Paris, 1847, 536-538.

[9] F. Feingold, D. Spohn: Les problèmes de répartition de puissance dans les réseaux maillés à courant alternatif. Analyse et méthodes numériques. Revue générale de l'électricité, 76(1976), 681-696.

[10] R. Narasimhan: Analysis on real and complex manifolds. Masson, Paris and NorthHolland, Amsterdam, 1968.

[11] J. Ortega, W. Rheinboldt: Iterative solution of nonlinear equations in several variables. Academic Press, New York, 1970. 


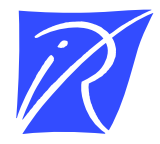

Unité de recherche INRIA Lorraine, Technopôle de Nancy-Brabois, Campus scientifique,

615 rue du Jardin Botanique, BP 101, 54600 VILLERS LÈS NANCY

Unité de recherche INRIA Rennes, Irisa, Campus universitaire de Beaulieu, 35042 RENNES Cedex

Unité de recherche INRIA Rhône-Alpes, 46 avenue Félix Viallet, 38031 GRENOBLE Cedex 1

Unité de recherche INRIA Rocquencourt, Domaine de Voluceau, Rocquencourt, BP 105, 78153 LE CHESNAY Cedex

Unité de recherche INRIA Sophia-Antipolis, 2004 route des Lucioles, BP 93, 06902 SOPHIA-ANTIPOLIS Cedex

Éditeur

INRIA, Domaine de Voluceau, Rocquencourt, BP 105, 78153 LE CHESNAY Cedex (France)

ISSN 0249-6399 